\title{
Do Amblyopic Eye First, Wait and Do Second Eye Cataract Surgery for Isoametropia, Accomodative Esotropic Amblyopia and Congenital Cataract Case
}

\author{
Goktug Demirci*, Gokhan Gulkilik, Mustafa Özsütçü, Banu Arslan, Mustafa Eliaçık \\ Department of Ophthalmology, Medipol University Hospital, Istanbul, Turkey \\ *Corresponding author: drgdemirci@yahoo.com
}

Received February 27, 2013; Revised March 27, 2013; Accepted April 07, 2013

\begin{abstract}
To review a case with isoametropic, accomodative esotropic amblyopia and congenital posterior polar cataract. A 12 years old child with visual acuities $2 / 10-7 / 10$, refractive error $+9,00 /+7,00$ and congenital cataract was observed previously for 5 years until the visual acuity of the right eye dropped 2 lines due to cataract in the more amblyopic eye. At first more amblyopic eye was operated and then we waited for 1 month to see how the amblyopic eye gains visual acuity. As the visual acuities become the same as $0,7 / 0,7$ other eye was operated. Do amblyopic eye first, wait and do second eye surgery was very successful in our patient. Visual acuities are $0,7 / 0,7$ and there is no accomodative esotropia. Conclusion: If there is a challenging case with congenital cataract, isoametropic amblyopia and accomodative esotropia together, performing cataract surgery to the amblyopic eye first and postponing the surgery of second eye for the regression of amblyopia could be a choice in this kind of patients.
\end{abstract}

Keywords: esotropia, congenital cataract, accommodation, strabismus

\section{Introduction}

Amblyopia is defined as the interruption of visual acuity, either unilaterally or bilaterally in spite of any refractive error being neutralized with lenses and in the absence of detectable eye disease the acuity of the eye is still below normal, which is resulting from a disturbance in retinal image formation caused by lack of stimuli or inappropriate or insufficient stimuli during the first decade of human life [1,2,3]. The estimated prevalence of amblyopia is $1-5 \%$ in the population [4,5]. It can be classified as strabismic, anisometropic, ammetropic or deprivational. Early treatment of amblyopia may result in improved vision, leading to a better prognosis for binocular vision development and a more stable alignment for surgery if required [6]. Animal models of unilateral visual deprivation have shown that few cortical neurons can be driven by the deprived eye and that, during an early critical period, these changes are reversible [7].

Accommodative esotropia is defined as a convergent ocular deviation associated with activation of the near synkinetic reflex to eliminate blurred retinal images produced by high hyperopic refractive errors. It usually presents in preschool years, and it is the most common type of strabismus, accounting for approximately one half of all childhood esotropia [8].

The purpose of this case report is to evaluate the results of sequential cataract extraction, posterior chamber intraocular lens (IOL) implantation done for a case with isoametropia, accomodative esotrophic strabismus, amblyopia and posterior polar cataract.

\section{Case}

Our case is a 12 yrs old female, who was diagnosed as isoametropia with a refractive error of $+9,00$ diopter right eye and +7,00 diopter left eye, accomodative esotrophic strabismus, posterior polar cataract, bilateral amblyopia with visual acuity of 0,4 right eye and 0,7 left eye examined by snellen acuity chart. While she was in follow-up annually in the paediatric ophthalmology section since she was 7 yrs old, her right eye visual acuity was gradually declined from 0,4 to 0,2 in following years but her left eye visual acuity did not change over time. This decline was expected to be due to posterior subcapsular cataract progression. We performed cataract surgery to the more amblyopic eye with implantation of 32,5 diopter Alcon IQ SN60WF IOL after the operation we did not do the surgery to the fellow eye, and we stopped her using glasses to left eye. According to our hypothesis this would act as a patching effect and so we waited until the visual acuity of amblyopic eye and fellow eye were equilised. This time period took 2 months after the first operation until the visual acuities equilised and we performed cataract surgery to the right eye with implantation of 31,5 diopter Alcon IQ SN60WF. Table 1. Follow up of visual acuities are shown in Figure 1.

\section{Discussion}

The traditional amblyopia therapy consists of patching or penalizing the fellow preferred eye, thus forcing the 
brain to use the visual input carried by the amblyopic eye [9]. Despite the belief that amblyopia is an untreatable pathology in adults, recent studies on animal models and clinical trials have challenged this picture, providing exciting evidence that intervention strategies boosting brain plasticity in adulthood may allow the reinstatement of visual functions in amblyopic subjects in any age [10]. This may be why our patient also gained two more lines of visual acuity even she is 12 yrs old.

Table 1. Visual acuities and time period from 3 yrs prior and 3 months after the IOL implantation.

\begin{tabular}{|c|c|c|}
\hline TIME & R EYE & L EYE \\
\hline $10 / 06 / 2009$ & 0,4 & 0,7 \\
\hline $07 / 07 / 2010$ & 0,4 & 0,7 \\
\hline $02 / 05 / 2011$ & 0,4 & 0,7 \\
\hline $06 / 09 / 2012$ & 0,2 & 0,6 \\
\hline 1 st month & 0,5 & 0,6 \\
\hline 2nd month after opr & 0,7 & 0,6 \\
\hline After Fellow eye is operated & 0,7 & 0,7 \\
\hline
\end{tabular}

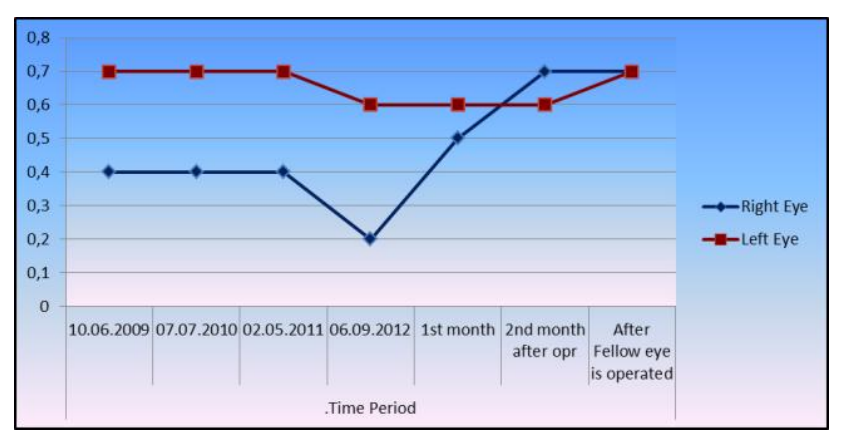

Figure 1. Follow up of visual acuities and time period from 3 yrs prior and 3 months after the IOL implantation.

Accommodation can lead to excessive convergence, which eventually exceeds the fusional divergence amplitude, resulting in esotropia. Refractive accommodative esotropia is characterized by high hyperopia, moderate angle of esodeviation and normal accommodative convergence to accommodation ratio $[11,12]$. It has a very favorable prognosis if the appropriate treatment is initiated promptly. Traditional methods of treating accommodative or partially accommodative esotropia include spectacle or contact lens correction of the hyperopic refractive error. Approximately 50\% of all pediatric esotropias are either entirely or partly accommodative and correction of high hypermetropia with glasses is the primary treatment for accommodative esotropia [13]. Prescription of the full amount of hyperopic correction provides adequate treatment for accommodative esotropia in $75 \%$ of cases [14]. Unfortunately these glasses are often poorly accepted by adolescents and young adults for they are cosmetically undesirable, heavy, and esotropia occurs with their removal.

Surgical attempts to correct hyperopia have yielded varying results over the last 130 years. These techniques include the reshaping of the cornea through incisions, burns, or lamellar cuts with removal of peripheral tissue; the addition of central inlays; laser ablations; and the replacement of the crystalline lens. Laser-assisted in situ keratomileusis (LASIK) and photorefractive keratectomy
(PRK) are the most commonly used keratorefractive procedures $[15,16,17,18]$. Kirwan $\mathrm{C}$ et al used Laser in situ keratomileusis, laser epithelial keratomileusis and Artisan phakic intraocular lens implantation and was successful especially in accomodative esotropias [19].

We first tried to treat accomodative esotrophia of our patient with glasses and we were successful in doing this. Although some ophthalmologists also suggest refractive surgery in these cases, unfortunately the progression of cataract led us do the surgical procedure as a clear lens extraction. If there was no cataract we would also choose the other refractive surgery procedures to allow her using her accomodative power until she gets presbiyobic. Spectacle correction of extreme myopic or hyperopic refractive errors can lead to prismatically induced optical aberrations especially in peripheral gaze, which can result in a narrow field of vision, and can subject the pediatric patient to social discomfort. Furthermore, spectacleinduced aniseikonia can impede the development of binocular vision [7,20]. Yassine et Al. suggests that for myopia of more than $12 \mathrm{D}$ or hyperopia of more than $5 \mathrm{D}$, IOL implantation may be advocated [7]. In our case we implanted Alcon monofocal IOL but because IOL tilt and posterior capsular opacification risks were more seen in this age group, we have choosed the simple but the safe way by not using a multifocal IOL. Multifocal IOLs are more sensitive to decentration, which is more common in pediatric cataract surgery, to prevent her from future IOL exchange procedure which would be risky because of general anesthesia and also possible refractive surgery risks [21].

Uncorrected anisohyperopia of more than 4.0 D causes amblyopia in $100 \%$ of the cases [22]. The mainstay of amblyopia treatment is providing a clear retinal image for the amblyopic eye if deprivation exists; however, merely removing the initial deprivation condition is usually insufficient to reverse the amblyopia, which is not true in our case; The second step consists of occluding or penalizing the dominant eye. As in the literature most effective is patching the good eye, unfortunately this age group is not aware of their illness and do not let the parents patch their eyes for an adequate time. Thus in our case we did not patched the good eye instead relatively decreased its vision for a period of time by operating it a little bit later and not wearing the glasses at that period of time. At the end of second month the visual acuity of bad eye was the same with the good eye.

As a conclusion in the light of our experience in cases with isoametropic ambliopia, congenital cataract and accomodative esotropia clear lens extraction and IOL implantation could be asuitable and safe treatment choice.

\section{References}

[1] Tongue AC, "Refractive errors in children," Pediatr Clin North Am, 34. 1425-37. 1987.

[2] Friendly DS. "Amblyopia: definition, classification, diagnosis, and management considerations for pediatricians, family physicians, and general practitioners," Pediatr Clin North Am.; 34. 13891401.1987.

[3] Flom MC, Neumaier RW. "Prevalence of amblyopia," Public Health Rep. 81. 329-41.1966.

[4] Holmes JM, Clarke MP. "Amblyopia." Lancet. 22; 367(9519):1343-51. Apr 2006. 
[5] Holmes JM, Repka MX, Kraker RT, Clarke MP. "The treatment of amblyopia. " Strabismus. 14(1):37-42. Mar 2006.

[6] Birch EE, Stager DR Sr, Wang J, O'Connor. "A.Longitudinal changes in refractive error of children with infantile esotropia." Eye. 24(12):1814-21. Dec 2010.

[7] Daoud YJ, Hutchinson A, Wallace DK, Song J, Kim T. "Refractive surgery in children: treatment options, outcomes, and controversies. " Am J Ophthalmol. 147(4):573-82. Apr 2009.

[8] Mohney BG. "Common forms of childhood esotropia, "Ophthalmology.;108(4):805-9 Apr 2001.

[9] Wu C, Hunter DG. "Amblyopia: diagnostic and therapeutic options. " Am J Ophthalmol. 141(1):175-84 Jan 2006.

[10] Baroncelli L, Maffei L, Sale A. "New perspectives in amblyopia therapy on adults: a critical role for the excitatory/inhibitory balance. " Front Cell Neurosci.; 5:25 2011.

[11] Mohan K, Sharma A. "Development of refractive accommodative esotropia in children initially diagnosed with pseudoesotropia". $J$ AAPOS.;16(3):266-8. Jun 2012.

[12] Kothari M, Manurung F, Paralkar S.Use of atropine to predict the accommodative component in esotropia with hypermetropia. Indian J Ophthalmol. 59(6):487-90 Nov-Dec 2011.

[13] Robert P. "Rutstein,Optometry" Volume 79, Issue 8 , Pages 422431 August 2008.

[14] Cho YA, Yi S, Kim SW. "Clinical evaluation of cessation of hyperopia in 123 children with accommodative esotropia treated with glasses for best corrected vision". Acta Ophthalmol:; 87(5):532-7. Aug 2009.

[15] Paysse EA, Tychsen L, Stahl E. "Pediatric refractive surgery: corneal and intraocular techniques and beyond. J AAPOS.; 16(3):291-7. Jun 2012.

[16] Paysse EA. "Unilateral lens extraction for high anisometropic myopia in children and adolescents: is this prudent?"J AAPOS.; 11(2):111-2. Apr 2007.

[17] Song J, Al-Ghamdi I, Awad A. "Pediatric refractive surgery in evolution".Middle East Afr J Ophthalmol.; 19(1):22-3. Jan 2012.

[18] Tychsen L "Refractive surgery for children: excimer laser, phakic intraocular lens, and clear lens extraction." Curr Opin Ophthalmol.; 19(4):342-8. Jul 2008.

[19] Kirwan C, O'Keefe M, O'Mullane GM, Sheehan C. "Refractive surgery in patients with accommodative and non-accommodative strabismus: 1-year prospective follow-up."Br J Ophthalmol.; 94(7):898-902. Jul 2010.

[20] Peterseim MW, Wilson ME. "Bilateral intraocular lens implantation in the pediatric population." Ophthalmology. 107(7):1261-6. Jul 2000.

[21] Jacobi PC, Dietlein TS, Jacobi FK. "Scleral fixation of secondary foldable multifocal intraocular lens implants in children and young adults." Ophthalmology; 109(12):2315-24. Dec 2002.

[22] Cruz OA, Scott WE, Davitt BV. "Isoametropic amblyopia due to high hyperopia in children." J AAPOS; 8:310-13. 2004. 